\title{
Eine selbstkonsistente Carleman Linearisierung zur Analyse von Oszillatoren
}

\author{
Harry Weber and Wolfgang Mathis \\ Institut für Theoretische Elektrotechnik, Leibniz Universität Hannover, 30167, Hannover, Deutschland \\ Correspondence to: H. Weber (weber@tet.uni-hannover.de)
}

Received: 1 December 2016 - Accepted: 20 February 2017 - Published: 21 September 2017

Kurzfassung. Die Analyse nichtlinearer dynamischer Schaltungen ist bis heute eine herausfordernde Aufgabe, da nur selten analytische Lösungen angegeben werden können. Daher wurden eine Vielzahl von Methoden entwickelt, um eine qualitative oder quantitative Näherung für die Lösungen der Netzwerkgleichung zu erhalten. Oftmals wird beispielsweise eine Kleinsignalanalyse mit Hilfe einer Taylorreihe in einem Arbeitspunkt durchgeführt, die nach den Gliedern erster Ordnung abgebrochen wird. Allerdings ist diese Linearisierung nur in der Nähe des stabilen Arbeitspunktes für hyperbolische Systeme gültig. Besonders für die Analyse des dynamischen Verhaltens von Oszillatoren treten jedoch nichthyperbolische Systeme auf, sodass diese Methode nicht angewendet werden kann (Mathis, 2000).

Carleman hat gezeigt, dass nichtlineare Differentialgleichungen mit polynomiellen Nichtlinearitäten in ein unendliches System von linearen Differentialgleichungen transformiert werden können (Carleman, 1932). Wird das unendlichdimensionale Gleichungssystem für numerische Zwecke abgebrochen, kann bei Oszillatoren der Übergang in eine stationäre Schwingung (Grenzzyklus) nicht wiedergegeben werden.

In diesem Beitrag wird eine selbstkonsistente Carleman Linearisierung zur Untersuchung von Oszillatoren vorgestellt, die auch dann anwendbar ist, wenn die Nichtlinearitäten keinen Polynomen entsprechen. Anstelle einer linearen Näherung um einen Arbeitspunkt, erfolgt mit Hilfe der Carleman Linearisierung eine Approximation auf einem vorgegebenen Gebiet. Da es jedoch mit der selbstkonsistenten Technik nicht möglich ist, das stationäre Verhalten von Oszillatoren zu beschreiben, wird die Berechnung einer PoincaréAbbildung durchgeführt. Mit dieser ist eine anschließende Analyse des Oszillators möglich.

\section{Einleitung}

Die Analyse elektrischer Schaltungen mit Hilfe von linearen Schaltungsmodellen ist bis heute ein fundamentales Konzept (Chua et al., 1987). Allerdings lassen sich einige auftretende Effekte in elektrischen Schaltungen nur durch nichtlineare Modelle korrekt wiedergeben. Dazu gehören unter anderem Oszillatoren oder PLL-Schaltungen, deren dynamisches Verhalten nur unter Berücksichtigung der Nichtlinearität beschrieben werden kann (Mathis, 2000). Für den Aufbau von integrierten elektrischen Oszillatoren werden neben Widerständen, Induktivitäten und Kapazitäten zusätzlich Halbleiterbauelemente verwendet, deren Verhalten auf Basis der Festkörperphysik beschrieben wird. Dadurch treten eine Vielzahl von nichtlinearen Funktionen, wie beispielsweise Exponential-, Polynomial- oder Wurzelfunktionen, in der Beschreibung des elektrischen Netzwerkes auf (Tsividis and Andrew, 2011). Werden zusätzlich noch dynamische Netzwerkelemente verwendet, lässt sich durch Anwendung der Kirchhoffschen Regeln ein nichtlineares Differentialgleichungssystem zur Beschreibung des elektrischen Netzwerkes aufstellen. Allerdings existiert nur in den seltensten Fällen eine analytische Lösung. Nur für den Fall eines linearen, zeitinvarianten Netzwerkes, wird ein System von linearen Differentialgleichungen mit konstanten Koeffizienten erhalten, das im Allgemeinen analytisch gelöst werden kann.

Nichtlineare Differentialgleichungen können mit einer Vielzahl von Methoden durch lineare Differentialgleichungen approximiert werden. Eine übliche Methode ist die Anwendung der Taylorreihe in einem Arbeitspunkt, die nach den Gliedern erster Ordnung abgebrochen wird. Mit Hilfe der Linearisierung kann eine Beschreibung des dynamischen Verhaltens in der Nähe des Arbeitspunktes nur für hyperbolische Systeme erfolgen. Bei der Analyse von Oszillatoren tritt jedoch ein nicht-hyperbolisches System auf, sodass nach 
dem Theorem von Hartman und Grobman das lineare System nicht zur Beschreibung in der Nähe des Arbeitspunktes verwendet werden kann (Guckenheimer and Holmes, 2002).

Eine weitere Möglichkeit, ein nichtlineares Gleichungssystem in ein lineares System zu überführen, bietet die Carleman Linearisierung. Carleman zeigte, dass gewöhnliche Differentialgleichungen bestehend aus Polynomen, in ein System von linearen Differentialgleichungen überführt werden können (Carleman, 1932). Da jedoch oftmals Halbleiterbauelemente in elektrischen Netzwerken verwendet werden, muss zunächst eine Transformation der nichtlinearen Differentialgleichung in ein polynomielle Differentialgleichung erfolgen. Anstelle einer Taylorreihe kann in diesem Fall die in der Physik bekannte Methode von Kerner angewendet werden, die eine äquivalente Transformation zwischen nichtlinearer und polynomieller Differentialgleichung ermöglicht (Kerner, 1981).

Bei der Carleman Linearisierung ist jedoch $\mathrm{zu}$ beachten, dass das äquivalente lineare System unendlichdimensional ist, sodass das unendlichdimensionale System in ein endlichdimensionales System, im einfachsten Fall durch Abschneiden nach einer vorgegebenen Dimension, überführt werden kann. Allerdings kann bereits anhand von einfachen Beispielen gezeigt werden, dass die Näherung nur in der Nähe des Anfangswertes gültig ist (Weber and Mathis, 2016). In diesem Beitrag wird daher zunächst eine selbstkonsistente Carleman Linearisierung beschrieben, die eine Erweiterung der abgeschnittenen Carleman Linearisierung darstellt. Diese Erweiterung erlaubt es, das dynamische Verhalten des nichtlinearen Systems auf einem vorgegebenen Gebiet zu beschreiben und damit eine Approximation über einen längeren Zeitraum gegenüber der abgeschnittenen Carleman Linearisierung zu erreichen.

Da jedoch mit der selbstkonsistenten Technik nur eine Näherung des dynamischen Oszillatorverhaltens über ein endliches zeitliches Intervall möglich ist, kann keine Analyse des stationären Zustands durchgeführt werden. Allerdings lässt sich mit Hilfe der Approximation der selbstkonsistenten Carleman Linearisierung eine Poincaré-Abbildung berechnen, mit der eine anschließende Analyse des stationären Zustands durchgeführt werden kann.

In Abschnitt 2 wird zunächst eine kurze Einführung in die Carleman Linearisierung gegeben und die Transformation nichtlinearer Differentialgleichung in polynomielle Differentialgleichung beschrieben. Anschließend wird in Abschnitt 3 die selbstkonsistente Carleman Linearisierung vorgestellt und an einem Beispiel präsentiert. Die Analyse mit Hilfe der selbstkonsistenten Carleman Linearisierung durch Anwendung einer Poincaré-Abbildung wird in Abschnitt 4 erläutert. Abschließend wird in Abschnitt 5 eine Anwendung präsentiert.

\section{Gewöhnliche Differentialgleichungen und Carleman Linearisierung}

Die Carleman Linearisierung erlaubt es, eine Differentialgleichung mit einem polynomiellen Vektorfeld $\boldsymbol{F}: \mathbb{R}^{k} \rightarrow \mathbb{R}^{k}$

$\frac{\mathrm{d} \boldsymbol{x}}{\mathrm{d} t}=\boldsymbol{F}(\boldsymbol{x})=\mathbf{A}_{0}+\mathbf{A}_{1} \boldsymbol{x}+\mathbf{A}_{2} \boldsymbol{x}^{[2]}+\cdots+\mathbf{A}_{l} \boldsymbol{x}^{[l]}$

mit

$x^{[l]}:=\underbrace{x \otimes x \ldots \otimes x}_{l \text {-mal }}$

wobei $\otimes$ das Kroneckerprodukt darstellt, und dem Anfangswert $\boldsymbol{x}(0)$ in ein unendlichdimensionales lineares Differentialgleichungssystem

$\dot{\boldsymbol{x}}=\mathbf{A} \boldsymbol{x}$,

mit der unendlichen Matrix $\mathbf{A} \in \mathbb{R}^{\infty} \times \mathbb{R}^{\infty}$ und dem unendlichen Vektor $\boldsymbol{x}$ zu überführen. Zur besseren Veranschaulichung wird der eindimensionale Fall $F: \mathbb{R} \rightarrow \mathbb{R}$

$\frac{\mathrm{d} x}{\mathrm{~d} t}=F(x)=\sum_{i=0}^{l} \alpha_{i} x^{i}=\alpha_{0}+\alpha_{1} x+\alpha_{2} x^{2}+\cdots \alpha_{l} x^{l}$

betrachtet.

Die Transformation von (4) in (3) erfolgt abhängig des Bildbereichs $\Omega:=\operatorname{range}(x)$ von $x: t \longmapsto x(t)$, sodass eine Abschätzung des Bildbereichs, beispielsweise mit Hilfe einer numerischen Integration, vorher erfolgen muss. Dabei werden folgende drei Fälle unterschieden, der Bildbereich entspricht (i) der vollständigen reellen Achse $\Omega=\mathbb{R}$, (ii) dem endlichen Intervall $\Omega=[a, b] \subset \mathbb{R}$, (iii) der positiven (negativen) Halbachse $\Omega=\mathbb{R}^{+(-)}$.

Es werden Polynome auf $\Omega$ betrachtet, die eine Orthonormalbasis des entsprechenden Polynomraums (Hilbertraum) auf $\mathbb{R}$ bilden, wenn eine geeignete Wichtungsfunktion $w: \mathbb{R} \rightarrow \mathbb{R}^{+}$für $\Omega$ verwendet wird, um ein Skalarprodukt

$(\Psi, \Phi):=\int_{\Omega} \Psi(x) \Phi(x) w(x) \mathrm{d} x$

zu definieren, sodass $\sqrt{(\Psi, \Psi)}=\|\Psi\|<\infty$ gilt (Rudin, 1987). In Abhängigkeit von $\Omega$ bilden die orthonormalen Polynome Lösungen eines Sturm-Liouville Problems (Lesky, 1962). Beispielsweise entsprechen für den Fall $\Omega=[a, b]$ die Lösungen den Jacobi-Polynomen. In Tabelle 1 sind abhängig von $\Omega$ die jeweiligen Polynome und die zugehörigen Wichtungen $w(x)$ angegeben. Zusätzlich zu den reellwertigen Polynomen (Jacobi-, Laguerre- und Hermite-Polynome), gibt es die komplexen orthonormalen Polynome, die einer äquivalenten komplexen Darstellung der Hermite-Polynome für den Fall $\Omega=\mathbb{R}$ entsprechen (Kowalski, 1997). 
Tabelle 1. Orthonormalen Polynome in Abhängigkeit von $\Omega$ (Kowalski, 1997).

\begin{tabular}{lll}
\hline Intervall & Polynom & Wichtungsfunktion \\
\hline$a \leq x \leq b$ & Jacobi-Polynome & 1 \\
\hline $\begin{array}{l}a \leq x \leq a \\
a \leq x\end{array}$ & Laguerre-Polynome & $\mathrm{e}^{-|x|}$ \\
\hline$-\infty \leq x \leq \infty$ & Hermite-Polynome & $\mathrm{e}^{-x^{2}}$ \\
\hline
\end{tabular}

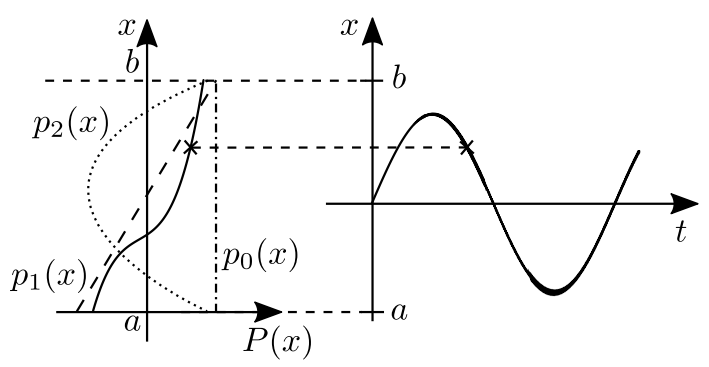

Abb. 1. Darstellung einer Funktion $P(x)$ mit Jacobi-Polynomen $p_{n}(x)$ auf $\Omega=[a, b]$.

Im Weiteren werden die orthonormalen Polynome mit $p_{n}(x)$ bezeichnet, wobei $n$ den höchsten Grad des Polynoms angibt. Für die Polynome $p_{n}(x)$, die auf $\Omega$ definiert sind, gilt

$\left(p_{n}, p_{m}\right)=\delta_{n m}$

mit dem Kronecker-Delta

$\delta_{n m}=\left\{\begin{array}{ll}1 & \text { mit } n=m \\ 0 & \text { mit } n \neq m\end{array}\right.$.

Wird eine Funktion $P(x)$ auf $\Omega$ betrachtet, kann diese nach dem Satz von Weierstrass durch eine Linearkombination der entsprechenden orthonormalen Polynome approximiert werden (Rudin, 1987). In Abb. 1 ist dies beispielhaft für $\Omega=$ $[a, b]$ mit Jacobi-Polynomen dargestellt.

Mit (4) kann die zeitliche Ableitung von $p_{n}(x)$ berechnet werden. Durch Anwendung der Kettenregel folgt für die Ableitung der Polynome $n=(0,1,2, \ldots)$

$$
\begin{aligned}
\frac{\mathrm{d} p_{n}(x)}{\mathrm{d} t} & =\frac{\mathrm{d} x}{\mathrm{~d} t} \frac{\mathrm{d} p_{n}(x)}{\mathrm{d} x}=F(x) \frac{\mathrm{d} p_{n}(x)}{\mathrm{d} x} \\
& =\left(\sum_{i=0}^{l} \alpha_{i} x^{i}\right) \frac{\mathrm{d} p_{n}(x)}{\mathrm{d} x} .
\end{aligned}
$$

In (8) ist zu erkennen, dass die Ableitung einem neuen Polynom vom Grad $l+(n-1)$ entspricht, da die Ableitung von $p_{n}(x)$ nach $x$ zu einem Polynom vom Grad $n-1$ führt. Die Ableitung in (8) kann daher als Linearkombination $(n=0,1,2, \ldots)$

$$
\frac{\mathrm{d} p_{n}(x)}{\mathrm{d} t}=\sum_{i=0}^{l+n-1} a_{n, i} p_{i}(x)
$$

dargestellt werden, wobei

$a_{n, i}=\left(F(x) \frac{\mathrm{d} p_{n}(x)}{\mathrm{d} x}, p_{i}\right)$

gilt. Gleichung (9) entspricht einem unendlichdimensionalen System von linearen Differentialgleichungen mit konstanten Koeffizienten, sowie den Anfangswerten $p_{n}(x(0))$, mit der entsprechenden Matrixform in (3), wobei A eine obere Dreiecksform besitzt (Kowalski and Steeb, 1991). Die Lösung des ursprünglichen nichtlinearen Differentialgleichungssystems in (4) erhält man mit $p_{1}(x)$, da alle orthonormalen Polynome $p_{1}(x)$ linear von $x$ abhängen und damit eine Rücktransformation ermöglicht wird. $\mathrm{Zu}$ beachten ist, dass sich der lineare Fall mit $l \leq 1$ in (4), auf ein endlichdimensionales lineares System reduziert.

Für den $k$-dimensionalen Fall in (1) kann der erweiterte Ansatz

$\Phi_{\mathbf{n}}(\boldsymbol{x})=\prod_{i=1}^{k} p_{n_{i}}\left(x_{i}\right) \quad$ mit $\mathbf{n}=\left(n_{1}, n_{2}, \cdots, n_{k}\right)$

verwendet werden (Steeb and Wilhelm, 1980).

\section{Transformation nichtlinearer Differentialgleichung in polynomielle Differentialgleichung}

Die Carleman Linearisierung kann nur auf polynomielle Differentialgleichung angewendet werden, wie in Abschnitt 2 dargestellt. Im Rahmen der Modellierung elektronischer Schaltungen treten jedoch auch nicht polynomielle Nichtlinearitäten auf, wie beispielsweise Exponential- oder Wurzelfunktionen. Um dennoch die Methode der Carleman Linearisierung anwenden zu können, nutzen wir eine wenig bekannte Methode von Kerner (1981). Mit dieser Methode können eine Vielzahl von nichtlinearen Differentialgleichungen in ein äquivalentes System von polynomiellen Differentialgleichungen überführt werden. Für die Transformation werden hierzu weitere Zustandsvariablen eingeführt, die den Nichtlinearitäten entsprechen. Hierdurch hat jedoch das polynomielle Differentialgleichungssystem gegenüber dem nichtlinearen Differentialgleichungssystem eine höhere Dimension. Zur besseren Veranschaulichung wird Kerners Konzept anhand des einfachen nichtlinearen Netzwerks in Abb. 2 illustriert. Die nichtlineare Induktivität kann bei einseitiger Aussteuerung und Beachtung von Sättigungseffekten durch

$i(\psi)=\alpha\left(\mathrm{e}^{\beta \psi}-1\right)$,

wobei $\psi$ der magnetischen Flussverkettung entspricht, approximiert werden (Philippow, 1971). Hierdurch können die Einschaltvorgänge des Netzwerkes mit der nichtlineare Differentialgleichung

$\frac{\mathrm{d} \psi}{\mathrm{d} t}+\alpha R\left(\mathrm{e}^{\beta \psi}-1\right)=U$ 


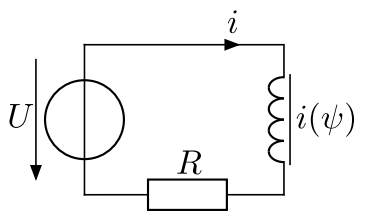

Abb. 2. Nichtlineares dynamisches Netzwerk (Philippow, 1971).

beschrieben werden. Durch Einführung der neuen Variable $x:=\mathrm{e}^{\beta \psi}$ und der Berechnung der Ableitung von $x$ nach der Zeit

$\frac{\mathrm{d} x}{\mathrm{~d} t}=\frac{\mathrm{d} \psi}{\mathrm{d} t} \frac{\mathrm{d} x}{\mathrm{~d} \psi}=(U-\alpha R(x-1)) \beta \mathrm{e}^{\beta \psi}$,

kann die nichtlineare Differentialgleichung in (13) in die äquivalente polynomielle Differentialgleichung

$\frac{\mathrm{d} x}{\mathrm{~d} t}=x(\beta \alpha R+\beta U)-\beta \alpha R x^{2}$

überführt werden. Der Fluss $\psi$ kann anschließend durch $\psi=\frac{1}{\beta} \ln x$ berechnet werden. Diese polynomielle Differentialgleichung kann im Gegensatz zu (13) mit der Carleman Linearisierung als unendlichdimensionales System von linearen Differentialgleichungen dargestellt werden.

\section{Selbstkonsistente Carleman Linearisierung}

Wie in Abschnitt 2 gezeigt, lässt sich eine nichtlineare Differentialgleichung mit polynomiellem Vektorfeld $F(x)$ mit Hilfe der Carleman Linearisierung in ein System von linearen Differentialgleichung unendlicher Dimension, wie in (3) dargestellt, überführen. Die Lösung von (3) lautet

$\boldsymbol{x}(t)=\mathrm{e}^{\mathbf{A} t} \boldsymbol{x}_{0}$,

wobei A eine unendlichdimensionale obere Dreiecksmatrix ist.

Im Allgemeinen ist es jedoch nicht möglich, das unendlichdimensionale System für numerische Zwecke zu verwenden. Aus diesem Grund wird das unendlichdimensionale System durch ein $N_{\max }$-dimensionales lineares System von Differentialgleichungen approximiert

$\dot{\tilde{x}}=\tilde{\mathbf{A}} \tilde{\boldsymbol{x}}$

wobei $\widetilde{\mathbf{A}}$ einer reellen $N_{\max } \times N_{\max }$ Matrix und $\widetilde{\boldsymbol{x}}$ einem $N_{\text {max }}$-dimensionalen Vektor entsprechen.

Dazu wird die Dimension des unendlichdimensionalen Systems auf eine endliche Dimension $N_{\max }$ begrenzt (Kowalski and Steeb, 1991). Daraus folgt, dass alle Koeffizienten $a_{n, i}$ in (9), mit $i>N_{\max }$, vernachlässigt werden, was zu einer Entkopplung führt. Hierdurch wird das endlichdimensionale Gleichungssystem $\left(n=0,1,2, \ldots, N_{\max }\right)$

$\frac{\mathrm{d} p_{n}(x)}{\mathrm{d} t} \approx \sum_{i=0}^{N_{\max }} a_{n, i} p_{i}(x)$ erhalten. Nach der Konstruktion in (18) führt die Carleman Linearisierung nur für Gleichgewichtspunkte der polynomiellen Differentialgleichung im Nullpunkt zu einer Approximation des stationären Verhaltens. Dazu muss gegebenenfalls eine nichtlineare Transformation des nichtlinearen Systems durchgeführt werden. Für instabile Gleichgewichtspunkte führt die Carleman Linearisierung zu einer Approximation in der Nähe des Anfangswertes. Weiterhin kann festgestellt werden, dass die Approximation für Anfangswerte in einer Umgebung des Ursprungs eine geringere Abweichung gegenüber der numerischen Lösung des nichtlinearen Systems aufweist.

Eine Alternative zu der Methode der abgeschnittenen Carleman Linearisierung stellt die selbstkonsistente Technik dar. Bei der selbstkonsistenten Carleman Linearisierung wird das Vektorfeld in (9), das aus Basisfunktionen $p_{i}(x)$ mit $i>N_{\max }$ besteht, mit Hilfe aller Basisfunktionen mit $i \leq N_{\text {max }}$ auf einem vorgegebenen Gebiet $\Omega_{1} \subset \Omega$ durch $\left(n=0,1,2, \ldots, N_{\max }\right)$

$\frac{\mathrm{d} p_{n}(x)}{\mathrm{d} t} \approx \sum_{i=0}^{N_{\max }} b_{n, i} p_{i}(x)$

approximiert, wobei die Koeffizienten $b_{n, i}$ mit der Methode der kleinsten Fehlerquadrate

$\min _{x \in \Omega_{1}}\left\|\sum_{i=0}^{l+n-1} a_{n, i} p_{i}(x)-\sum_{i=0}^{N_{\max }} b_{n, i} p_{i}(x)\right\|_{2}^{2}$

berechnet werden, siehe z.B. Griva et al. (2009). Mit dieser Methode ist es möglich, das Verhalten der ursprünglichen nichtlinearen Differentialgleichungen für ein vorgegebenes $\Omega_{1} \mathrm{zu}$ approximieren. Hierbei ist jedoch $\mathrm{zu}$ beachten, dass die Lösung $x(t)$ für alle $t$ in dem vordefinierten Gebiet $\Omega_{1}$ bleibt.

Die selbstkonsistente Carleman Linearisierung kann auch zur Analyse von Systemen verwendet werden, die einen Grenzzyklus besitzen. Hierbei ist allerdings $\mathrm{zu}$ beachten, dass das stationäre Verhalten nicht beschrieben wird, da es nicht möglich ist, den auftretenden Grenzzyklus mit einem endlichdimensionalen linearen System zu beschreiben. Allerdings erlaubt die selbstkonsistente Technik im Gegensatz zu der abgeschnittenen Carleman Linearisierung eine Approximation über mehrere Perioden, abhängig vom betrachteten System und dem Gebiet $\Omega_{1}$.

Die Unterschiede zwischen der abgeschnittenen und selbstkonsistenten Carleman Linearisierung werden anhand der van-der-Pol-Gleichung

$$
\begin{aligned}
& \frac{\mathrm{d} x_{1}}{\mathrm{~d} t}=x_{2} \\
& \frac{\mathrm{d} x_{2}}{\mathrm{~d} t}=\varepsilon x_{2}\left(1-x_{1}^{2}\right)-x_{1}
\end{aligned}
$$

mit den Anfangswerten $x_{1}(0)$ und $x_{2}(0)$ für $\varepsilon=\frac{1}{10}$ erläutert. In Abb. 3 sind die beiden Methoden im Vergleich zu der numerischen Lösung der nichtlinearen Differentialgleichung in 


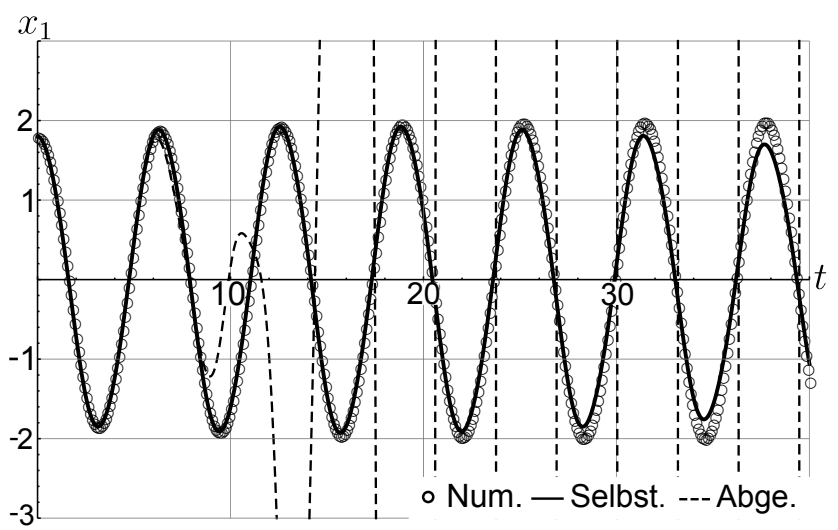

Abb. 3. Vergleich der abgeschnittenen (gestrichelte Linie) und selbstkonsistenten (durchgezogene Linie) Carleman Linearisierung mit der numerischen (Kreise) Lösung für die van-der-PolGleichung mit dem Anfangswert $x_{1}(0)=1,8$.

(21) dargestellt. Wie zu erkennen ist, führt das Abschneiden nach einer Dimension bereits nach kurzer Zeit zu einem instabilen Verhalten im Vergleich zu der numerischen Lösung. Die selbstkonsistente Carleman Linearisierung führt dagegen zu einer Approximation über einen längeren Zeitbereich. Das Verhalten weicht jedoch mit zunehmender Zeit von der numerischen Lösung ab, da der auftretende Grenzzyklus nicht abgebildet werden kann.

Allerdings kann mit der selbstkonsistenten Carleman Linearisierung eine Poincaré-Abbildung berechnet werden, mit der eine anschließende Untersuchung des stationären $\mathrm{Zu}$ stands möglich ist. Darauf wird im folgenden Abschnitt eingegangen.

\section{Oszillatoren und die selbstkonsistente Carleman Linearisierung}

Wie in Abschnitt 3 gezeigt, ist es mit der selbstkonsistenten Carleman Linearisierung nicht direkt möglich, Grenzzyklen, die bei Oszillatoren auftreten, zu approximieren. Allerdings kann unter Anwendung der selbstkonsistenten Technik eine Poincaré-Abbildung berechnet werden, mit der eine anschließende Analyse von Oszillatoren möglich ist.

Es sei $\boldsymbol{x}_{\boldsymbol{p}}$ ein Grenzzyklus eines Flusses $\boldsymbol{g}_{t}$ im $\mathbb{R}^{k}$ gegeben, der durch eine nichtlineare Differentialgleichung beschrieben wird. Weiterhin sei eine Fläche $\Sigma \subset \mathbb{R}^{n}$ im Phasenraum definiert, die $\boldsymbol{x}_{\boldsymbol{p}}$ schneidet und nicht tangiert. Der Schnittpunkt von $\boldsymbol{x}_{\boldsymbol{p}}$ und $\Sigma$ sei mit $\boldsymbol{p}$ gegeben, wie in Abb. 4 dargestellt, siehe z.B. Guckenheimer and Holmes (2002). Nun lässt sich für einen Anfangswert $\boldsymbol{q} \in \Sigma$ in der Umgebung von $\boldsymbol{p}$ die Poincaré-Abbildung

$\boldsymbol{P}(\boldsymbol{q}):=\boldsymbol{g}_{\tau}(\boldsymbol{q})$

definieren, wobei $\tau=\tau(\boldsymbol{q})$ die von $\boldsymbol{q}$ abhängige Zeit ist, nach welcher der Fluss $\boldsymbol{g}_{t}(\boldsymbol{q})$ wieder $\Sigma$ schneidet. Für $\boldsymbol{p}$ folgt ent-

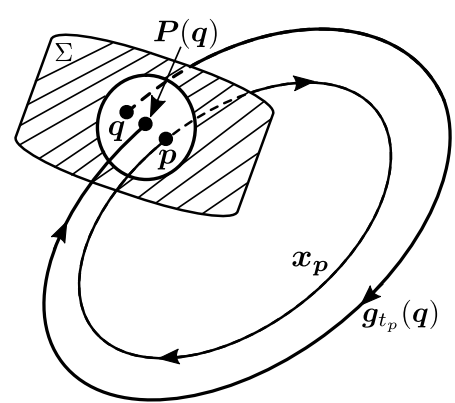

Abb. 4. Geometrische Interpretation der Poincaré-Abbildung (Guckenheimer and Holmes, 2002).

sprechend nach (22) die Fixpunktgleichung

$P(p)=p$.

Da die selbstkonsistente Carleman Linearisierung eine Approximation über mehrere Perioden ermöglicht, kann die Poincaré-Abbildung in (22) für Grenzzyklen $\boldsymbol{x}_{\boldsymbol{p}} \in \Omega_{1}$ berechnet werden. Dabei ist, wie in Abschnitt 3 gesagt, $\Omega_{1}$ geeignet zu wählen. Anschließend kann $\boldsymbol{p}$ in (23) bestimmt werden, sodass unter Anwendung der selbstkonsistenten Carleman Linearisierung der Grenzzyklus für eine Periode approximiert werden kann. Anhand der Approximation ist es anschließend möglich, eine Analyse des Oszillators durchzuführen.

Der beschriebene Ablauf wird anhand der in Abschnitt 3 angegebenen van-der-Pol-Gleichung illustriert.

Zur Berechnung der Poincaré-Abbildung wird zunächst $\Sigma$ im zweidimensionalen Phasenraum der vander-Pol-Gleichung definiert. In diesem Beispiel sei $\Sigma=$ $\left\{\left(x_{1}, x_{2}\right) \in \mathbb{R}^{2} \mid 0 \leq x_{1}, x_{2}=0\right\}$, d.h. positive Halbachse in $x_{1}$-Richtung, sodass für den Anfangswert auf $\Sigma$

$\boldsymbol{q}=\left(\begin{array}{c}x_{1}(0) \\ 0\end{array}\right)$

gilt. Durch Anwendung der selbstkonsistenten Carleman Linearisierung in Abschnitt 3, kann die Poincaré-Abbildung $P\left(x_{1}(0)\right)$ angegeben werden. Die Periode des Grenzzyklus bei der van-der-Pol-Gleichung in (21) entspricht dabei $\tau=2 \pi$. In Abb. 5 ist die Differenz der $\mathrm{Ab}$ bildung $P\left(x_{1}(0)\right)$ und $x_{1}(0)$ für verschiedene Anfangswerte dargestellt. Aus dieser Darstellung kann entnommen werden, ob der Schnittpunkt mit $\Sigma$ gegenüber dem Anfangswert zugenommen $\left(P\left(x_{1}(0)\right)-x_{1}(0)>0\right)$ oder abgenommen $\left(P\left(x_{1}(0)\right)-x_{1}(0)<0\right)$ hat. Der Fall $\left(P\left(x_{1}(0)\right)-x_{1}(0)=0\right)$ entspricht der Fixpunktgleichung in (23) und damit einem Punkt auf dem Grenzzyklus. Zusätzlich lässt sich anhand der negativen Steigung im Fixpunkt bei $x_{1}(0) \approx 2$ feststellen, dass es sich um einen stabilen Grenzzyklus handelt, da alle Anfangswerte durch mehrmalige Anwendung der Abbildung $P\left(x_{1}(0)\right)$ in den Fixpunkt konvergieren (Guckenheimer and Holmes, 2002). In Abb. 6 ist der 


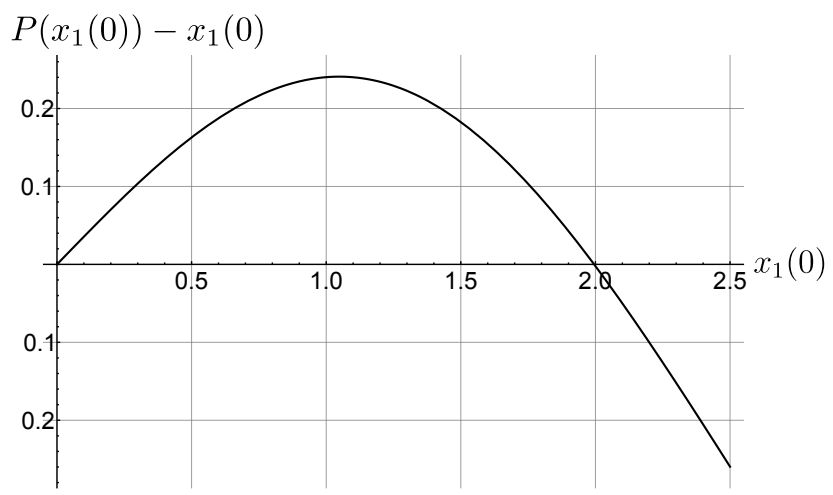

Abb. 5. Die Differenz zwischen der Poincaré-Abbildung und dem Anfangswert für die van-der-Pol-Gleichung.

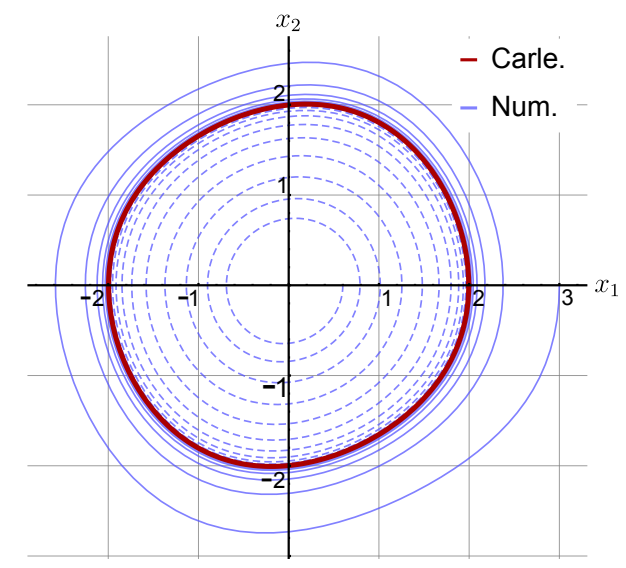

Abb. 6. Darstellung des approximierten Grenzzyklus mit der selbstkonsistenten Carleman Linearisierung (rote Linie) und zwei numerisch berechneter Trajektorien (blaue Linien) für die van-der-PolGleichung.

approximierte Grenzzyklus im Vergleich zu zwei numerisch berechneten Trajektorien dargestellt. Zur besseren Veranschaulichung wurde ein Anfangswert innerhalb (gestrichelte Linie) als auch außerhalb (durchgezogene Linie) des Grenzzyklus gewählt. Es ist zu erkennen, dass beide Trajektorien im Laufe der Zeit sich dem approximierten Grenzzyklus annähern. Wird schließlich der maximale Wert für $x_{1}$ und $x_{2}$ des Grenzzyklus innerhalb einer Periode bestimmt, kann die Amplitude der Oszillation angegeben werden. In Abb. 7 ist die numerische Lösung der van-der-Pol-Gleichung und die approximierte Amplitude mit der selbstkonsistenten Carleman Linearisierung dargestellt. Es ist zu erkennen, dass die Amplitude der Oszillation näherungsweise berechnet wird.

Es sei jedoch erwähnt, dass die Approximation bei beliebigen polynomiellen Systemen zu einem erhöhten Rechenaufwand führt. Wird beispielsweise eine sehr starke Nichtlinearität betrachtet $(\varepsilon \gg 1)$, muss eine entsprechende Erhöhung der Dimension $N_{\max }$ der selbstkonsistenten Carleman Linearisierung erfolgen, was wiederum zu einem erheblich größe-

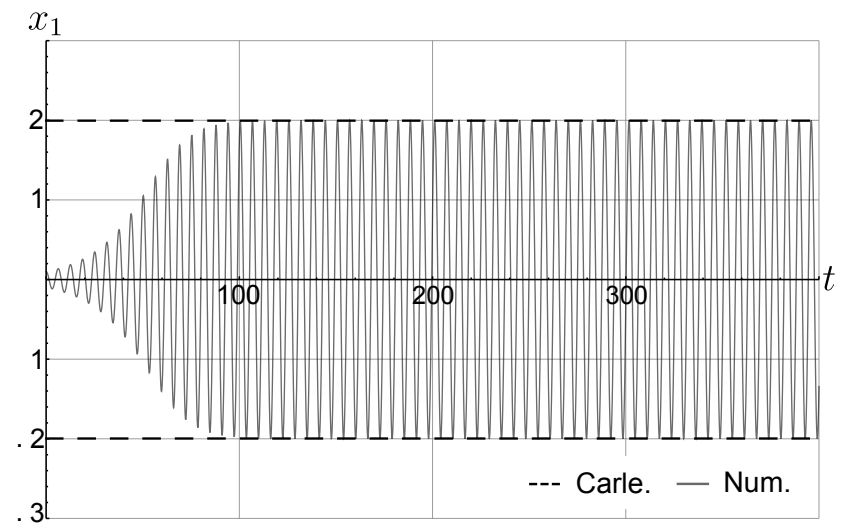

Abb. 7. Vergleich der abgeschätzten Amplitude von $x_{1}$ mit der selbstkonsistenten Carleman Linearisierung (gestrichelte Linie) und der numerischen Lösung (durchgezogene Linie) für die van-derPol-Gleichung.

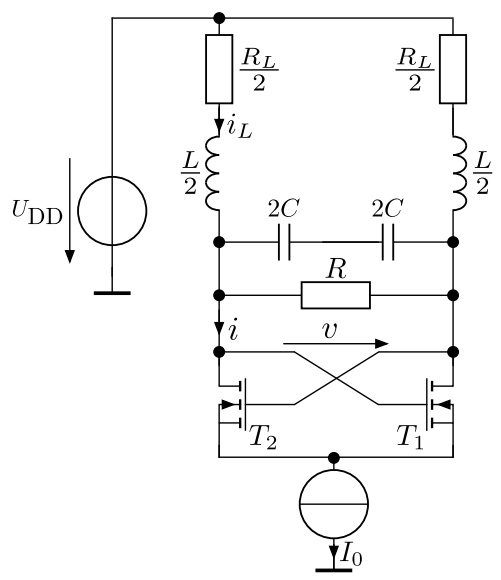

Abb. 8. Untersuchte Struktur einer Oszillatorschaltung.

ren rechnerischen Aufwand führt. Weiterhin muss darauf geachtet werden, dass die berechnete Poincaré-Abbildung nur in dem von der selbstkonsistenten Carleman Linearisierung begrenzten Bereich $\Omega_{1}$ gültig ist.

Im nächsten Abschnitt wird der in diesem Beitrag vorgestellte Ablauf zur Analyse von Oszillatoren bei einer LCTank Schaltung durchgeführt, die in heutigen integrierten Schaltungen eine breite Verwendung findet (Mathis and Bremer, 2011; Hegazi et al., 2005).

\section{Anwendungsbeispiel}

Eine besonders häufig eingesetzte Struktur einer Oszillatorschaltung stellt der LC-Tank Oszillator in Abb. 8 dar. Diese soll mit Hilfe der selbstkonsistenten Carleman Linearisierung analysiert werden. Das elektronische Netzwerk lässt sich für $-V_{\text {th }}<v<V_{\text {th }}$ durch die nichtlineare Differential- 
gleichung

$$
\begin{aligned}
\frac{\mathrm{d} i_{L}}{\mathrm{~d} t} & =\frac{v-i_{L} R_{L}}{2 L} \\
\frac{\mathrm{d} v}{\mathrm{~d} t} & =\frac{1}{C}\left(I_{0} \frac{v}{V_{n}} \sqrt{1-\frac{v^{2}}{V_{n}^{2}}}-i_{L}-\frac{v}{R}\right)
\end{aligned}
$$

mit $V_{n}=\alpha\left(I_{0}\right) \sqrt{\frac{4 I_{0} L_{T}}{\mu_{n} C_{\mathrm{Ox}} W_{T}}}$ beschreiben (Buonomo and Schiavo, 2004). Die Funktion $\alpha\left(I_{0}\right)$ dient zur Anpassung an den hier verwendeten Prozess für die Transistoren. Zur Transformation von (25) werden nacheinander die beiden neuen Variablen

$$
\begin{aligned}
& x_{3}:=\sqrt{1-\frac{v^{2}}{V_{n}^{2}}} \\
& x_{4}:=\frac{1}{x_{3}}=\frac{1}{\sqrt{1-\frac{v^{2}}{V_{n}^{2}}}}
\end{aligned}
$$

eingeführt, was zu dem polynomiellen Differentialgleichungssystem

$$
\begin{aligned}
\frac{\mathrm{d} i_{L}}{\mathrm{~d} t} & =\frac{v-i_{L} R_{L}}{2 L} \\
\frac{\mathrm{d} v}{\mathrm{~d} t} & =\frac{1}{C}\left(I_{0} \frac{v x_{3}}{V_{n}}-i_{L}-\frac{v}{R}\right) \\
\frac{\mathrm{d} x_{3}}{\mathrm{~d} t} & =\frac{-v x_{4}}{V_{n}^{2} C}\left(I_{0} \frac{v}{V_{n}} x_{3}-i_{L}-\frac{v}{R}\right) \\
\frac{\mathrm{d} x_{4}}{\mathrm{~d} t} & =\frac{v x_{4}^{3}}{V_{n}^{2} C}\left(I_{0} \frac{v}{V_{n}} x_{3}-i_{L}-\frac{v}{R}\right)
\end{aligned}
$$

führt. Anschließend wird die selbstkonsistente Carleman Linearisierung zur Berechnung der Poincaré-Abbildung nach Abschnitt 4 verwendet. In Abb. 9 ist der Vergleich zwischen der approximierten Amplitude durch die Carleman Linearisierung und der numerischen Lösung in Spectre Cadence dargestellt. Es ist zu erkennen, dass die approximierte Amplitude in guter Näherung mit der numerischen Lösung übereinstimmt.

\section{Zusammenfassung}

In diesem Beitrag wurde eine Methode zur Analyse von Oszillatoren mit einer selbstkonsistenten Carleman Linearisierung präsentiert. Für die Analyse wird zusätzlich zu der Carleman Linearisierung eine Poincaré-Abbildung verwendet, um eine Approximation des auftretenden Grenzzyklus durchführen zu können. Anschließend kann beispielsweise die Stabilität des Grenzzyklus untersucht werden oder die Amplitude der Oszillation berechnet werden. Dabei ist jedoch zu beachten, dass der vordefinierte Bereich $\Omega_{1}$ der selbstkonsistenten Carleman Linearisierung nicht verlassen wird. Außerdem ist zu beachten, dass abhängig von der

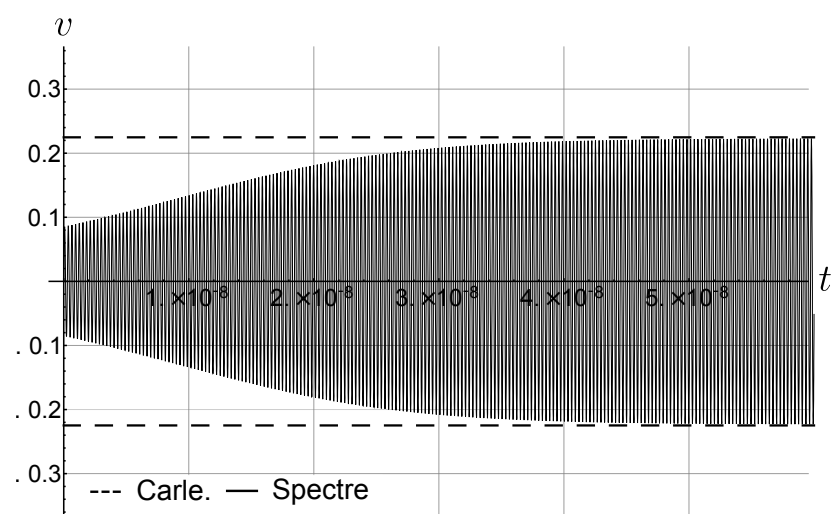

Abb. 9. Vergleich der numerischen Lösung des LC-Tank Oszillators in Cadence (durchgezogene Linie) und der approximierten Amplitude der selbstkonsistenten Carleman Linearisierung (gestrichelte Linie).

Nichtlinearität des Systems eine höhere Dimension $N_{\max }$ der Carleman Linearisierung verwendet werden muss, was zu einem erhöhten Anstieg des Rechenaufwands führt.

Interessenkonflikt. Die Autoren erklären, dass kein Interessenkonflikt besteht.

The publication of this article was funded by the open-access fund of Leibniz Universität Hannover.

Edited by: J. Anders

Reviewed by: B. Schlecker and one anonymous referee

\section{Literatur}

Buonomo, A. and Schiavo, A. L.: Modelling and analysyis of differential VCOs, International Journal of Circuits Theory and Applications, 32, 3, https://doi.org/10.1002/cta.270, 2004.

Carleman, T.: Application de la théorie des équations intégrales linéaires aux systèmes d'équations différentielles non linéaires, Acta Mathematica, 59, 1, https://doi.org/10.1007/BF02546499, 1932.

Chua, L. O., Desoer, C. A., and Kuh, E. S.: Linear and Nonlinear Circuits, McGraw-Hill Book Company, Singapur, 1987.

Griva, I., Nash, S. G., and Sofer, A.: Linear and Nonlinear Optimization: Second Edition, Siam, Philadelphia, 2009.

Guckenheimer, J. and Holmes, P.: Nonlinear Oscillation, Dynamical Systems and Bifurcations of Vector Fields, Springer, 2002.

Hegazi, E., Rael, J., and Abidi, A.: The Designer's Guide to HighPurity Oscillators, Kluwer Academic Publishers, Dordrecht, Netherlands, 2005.

Kerner, E. H.: Universal formats for nonlinear ordinary differential systems, J. Math. Phys., 22, 7, https://doi.org/10.1063/1.525074, 1981.

Kowalski, K.: Nonlinear dynamical systems and classical orthogonal polynomials, J. Math. Phys., 38, 5, https://doi.org/10.1063/1.531990, 1997. 
Kowalski, K. and Steeb, W.-H.: Nonlinear Dynamical Systems and Carleman Linearization, World Scientific, Singapur, 1991.

Lesky, P.: Die charakterisierung der klassischen orthogonalen polynome durch Sturm-Liouvillesche Differentialgleichungen, Arch. Ration. Mech. An., 10, 1, https://doi.org/10.1007/BF00281200, 1962.

Mathis, W.: Nonlinear electronic circuits - An overview, Proc. International Conference Mixed Design of Integrated Circuits and Systems, 2000.

Mathis, W. and Bremer, J.-K.: Design of nonlinear CMOS circuits in the Nano-GHz Era and its mathematical challenges, Math. Comput. Simulat., 82, 3, https://doi.org/10.1016/j.matcom.2010.10.013, 2011.

Philippow, E.: Nichtlineare Elektrotechnik, Akademische Verlagsgesellschaft, Leipzig, 1971.
Rudin, W.: Real and Complex Analysis, McGraw-Hill Book Company, Singapur, 1987

Steeb, W.-H. and Wilhelm, F.: Non-Linear Autonomous Systems of Differential Equations and Carleman Linearization Procedure, J. Math. Anal. Appl., 77, 2, https://doi.org/10.1016/0022247X(80)90250-4, 1980.

Tsividis, Y. and Andrew, C. M.: Operation and Modeling of the MOS Transistor, Oxford University Press, 2011.

Weber, H. and Mathis, W.: A Self-consistent Carleman Linearization Technique for the Large Signal Analysis of Nonlinear Circuits, IEEE International Symposium on Circuits and Systems (ISCAS 2016), https://doi.org/10.1109/ISCAS.2016.7538984, 2016. 